\title{
Effectiveness of probiotics in irritable bowel syndrome: methodological quality of meta-analyses and systematic reviews
}

Original article

Yuan Jiaa, Li-Min Guo ${ }^{a}$, Si-Yuan Yang ${ }^{a}$, Qian Wuª, Fan-Jie Meng ${ }^{\mathrm{b}, *}$

${ }^{a}$ Graduate College, Tianjin University of Traditional Chinese Medicine, Tianjin 301617, China

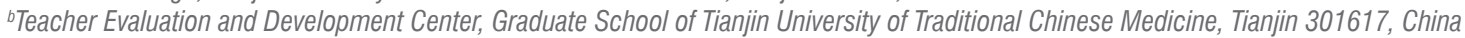

Received: 19 September 2018; Accepted: 27 December 2018; Published: 20 June 2019

Abstract: Objective: This study assessed the methodological quality of systematic reviews/meta-analysis of the effectiveness of probiotics against irritable bowel syndrome (IBS) using the accepted methodological quality assessment scale AMSTAR and explored the factors that influenced the quality of methodology. It was designed to provide a reference for future research and systematic reviews/metaanalysis.

Methods: The methodological quality of existing systematic reviews/meta-analysis was evaluated using the AMSTAR scale. Influencing factors of methodological quality were statistically analyzed using RevMan 5.3 software. The included systematic reviews/metaanalysis must include the following characteristics: (1) methods using systematic evaluation/meta-analysis, (2) probiotic intervention, and (3) language limitation to Chinese and English.

Results: The AMSTAR score was $5-9(7.42 \pm 1.22)$, and the quality is above average. The factors affecting the methodological quality are the number of authors and whether they cooperate with the institution.

Conclusions: Studies have shown that current systematic reviews/meta-analysis of the effectiveness of probiotics on IBS does not fully comply with methodological quality standards, and therefore the methodological quality of research in this area needs to be strengthened. To better clarify how probiotics affect IBS, future systematic reviews and meta-analyses should not only follow methodological quality standards but also include more effective outcome measures, and they should focus more on the discussion of research results. We look forward to the development of higher-quality randomized controlled trials in the future.

Keywords: probiotics • irritable bowel syndrome • systematic review • meta-analysis • methodological quality

(c) Shanxi Medical Periodical Press.

\section{Introduction}

Irritable bowel syndrome (IBS) is one of the most frequent digestive tract disorders, which is encountered by general practitioners and gastroenterologists. It is a functional disorder of the digestive tract, which causes chronic symptoms, such as abdominal pain and discomfort and abnormal bowel movements, including diarrhea and constipation, with no organic abnormalities. Prevalence of IBS varies between Asian and North American societies, but the total range in the general population is estimated to be $5 \%-11 \% .{ }^{1-3}$ Besides the interference with daily lives of patients

How to cite this article: Jia Y, Guo L-M, Yang S-Y, Wu Q, Meng F-J. Effectiveness of probiotics in irritable bowel syndrome: methodological quality of meta-analyses and systematic reviews. Front Nurs. 2019; 2: 1-7. 
and caregivers, socioeconomic costs of IBS have increased, as the majority of IBS patients are young (20-39 years). Although the exact pathophysiology underlying IBS is still not fully understood, chronic lowgrade mucosal inflammation, alterations in gut epithelial and immune function, and visceral hypersensitivity caused by alterations in intestinal microbiota have been shown to be associated with IBS. ${ }^{4-6}$

Pharmacological, psychological, and complementary approaches are considered as therapeutic options for IBS patients. Pharmacological medications include antispasmodics, selective serotonin reuptake inhibitors, tricyclic antidepressants, and 5-hydroxytryptamine type-3 (5-HT3) antagonists such as ramosetron and alosetron, as well as lubiprostone and linaclotide. However, IBS patients often have a variant response to these therapies, and also they are associated with several complications. ${ }^{7}$ Antidepressant treatment often causes severe problems, including weight gain, and it cannot be tolerated by many patients. Spasmolytics and 5-HT3 antagonists are ineffective in some cases, and they may even worsen the symptoms of IBS. ${ }^{8}$ Hence, many IBS patients are looking for other treatments, such as herbal medicine and Chinese acupuncture. Meanwhile, the long-term use of new therapeutic options with the potential to alter intestinal microbiota has recently been identified and includes the low fermentable, oligo-, di-, monosaccharides, and polyols (FODMAP) diet, ${ }^{9}$ antibiotics, ${ }^{10}$ and probiotics. Probiotics, defined as "live microorganisms that, when administered in adequate amounts, confer a health benefit on the host,"11 have the potential to influence the intestinal microbiota. Probiotics may affect intestinal barrier function and exert anti-inflammatory actions. ${ }^{12}$ To date, many clinical studies have investigated the effects of probiotics in IBS patients, and these studies demonstrated that probiotic administration is effective in IBS patients.

In this article, we summarize the systematic reviews and meta-analysis of the effectiveness of probiotics on IBS, evaluate the quality of the report and methodology, explore the influencing factors, providing strong evidence for the development of related work, and regulate the research process and methods in this field.

\section{Methods}

\subsection{Search strategy and study selection}

The literature search was carried out by two reviewers, and at the following electronic databases were searched: PubMed, The Cochrane Library, China National Knowledge Infrastructure (CNKI), Wangfang database, VIP database (from database establishment to June 2018). The final search term was "probiotics
OR probiotics preparation OR active microorganism OR lactic acid bacteria OR Bifidobacterium OR yeast OR tyrosine OR actinomycete OR actinomycetes AND intestinal irritable bowel syndrome OR IBS AND systematic reviews OR meta analysis."

Literature search included three steps: (1) Retrieval in the Chinese and English databases of the CNKI, Wanfang, VIP, PubMed, Embase, Web of Science, The Cochrane Library, etc. Literature, analysis of the titles, abstracts, keywords used, and subject words of the documents were obtained to further determine the keywords of the literature search. (2) All relevant keywords for database search were used. If the abstract met the inclusion criteria, there was a further search and complete look at the full text. (3) Through the reference to the literature obtained for additional search. The literature screening process is illustrated in Figure 1.

\subsection{Inclusion and exclusion criteria}

Studies were considered eligible if they met the following criteria: (1) if the studies were systematic reviews and meta-analysis that compared probiotics with placebo; (2) if the diagnosis of IBS was made according to the Rome III or Rome II criteria; (3) if the treatment duration was $>7$ days; and (4) if the dichotomous data on the overall syndrome response to the therapy or continuous score data of the effect on individual IBS symptoms or quality of life (QoL) could be extracted or obtained from the authors. Exclusion criteria were as follows: (1) studies with only an abstract; (2) studies in which probiotics were mixed with other drugs; (3) unable to obtain full text; and (4) studies in which the control group received probiotics.

\subsection{Literature screening and data extraction}

In accordance with the inclusion and exclusion criteria, two researchers screened the literature independently and read the full text with the help of Windows Excel 2010, including the author, year, country/region, number of studies, outcome indicators, etc. Documents published repeatedly with the same content shall be selected as journal papers published publicly and peer reviewed. Excel 2010 software was used to establish the data extraction form.

\subsection{Quality evaluation}

AMSTAR $^{13}$ has good validity, reliability, and responsiveness in evaluating the methodological quality of systematic evaluation/meta-analysis. In a total of 11 entries, each item was rated as "yes" 1 point, "part is" 0.5 points, "no" 0 points, score $0-4$ points were considered low quality, 5-8 medium quality, 9-11 high 


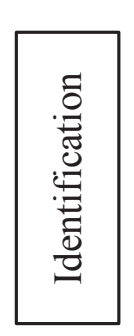

122 potential relevant studies identified

- 91 PubMed

- 8 The Cochrane Library

- 4 CNKI

- 3 VIP Database
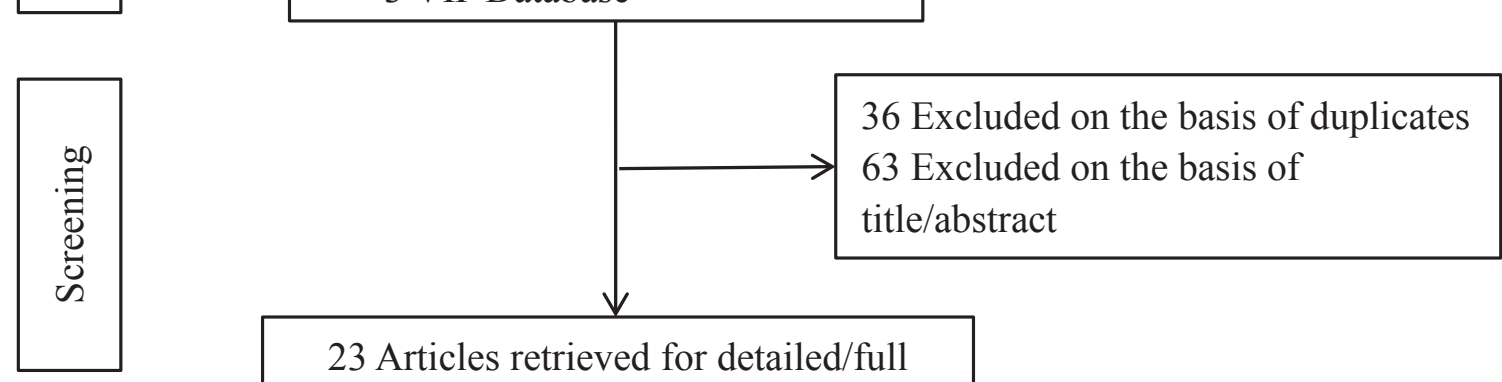

23 Articles retrieved for detailed/full

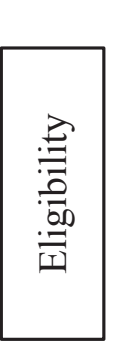
review

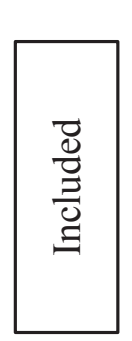

10 Excluded:

- 4 Failed to get full text

- 6 Protocol/Guidelines

Figure 1. Literature screening flowchart.

quality. Before the data extraction and quality evaluation, we first trained and pre-evaluated the use of the scale, discussed the possible problems in the process of data extraction and evaluation, and oobtained the data extraction after the agreement was obtained. The formal quality assessment process was completed by two researchers independently and cross-checked. If there was a disagreement, the formal quality assessment process could be resolved or decided by a third party.

\subsection{Statistical analysis}

In this study, the quality of meta-analysis was analyzed in two aspects, including the number of authors (less than two people vs. or more than three people) and institutions (multi-organization cooperative vs. single organization) to explore the factors affecting the quality of the analysis. The categorical data were compared by Chi-square test, the continuous data were compared by $t$-test, and the significance level was set at $\alpha=0.05$. All data were entered into Excel 2010 software, and RevMan 5.3 software was used for statistical analysis.

\section{Results}

\subsection{Literature search results}

Thirteen articles ${ }^{14-26}$ were included in the study. The general conditions for inclusion in the study are presented in Table 1. 


\begin{tabular}{|c|c|c|c|c|}
\hline First author & Year & Country & Studies included & Outcome indicators \\
\hline Corbitt et a $\left.\right|^{14}$ & 2018 & Australia & 24 & (1) (2) \\
\hline Ortiz-Lucas $\mathrm{M}^{15}$ & 2013 & Spain & 10 & (1) (2) (3) \\
\hline Didari $T^{16}$ & 2015 & Iran, China & 15 & (1) (2) \\
\hline Zhang $Y^{17}$ & 2016 & China & 21 & (1) (2) (3) (4) \\
\hline Yuan $\mathrm{F}^{18}$ & 2017 & USA & 5 & (1) (2) \\
\hline Ford A C ${ }^{19}$ & 2014 & USA, UK, Canada & 43 & (1) (2) \\
\hline Tiequn $\mathrm{B}^{20}$ & 2015 & China & 6 & (1) (2) (4) \\
\hline $\mathrm{Li} \mathrm{DQ}^{21}$ & 2016 & China & 5 & (1) (2) (4) (5) (6) \\
\hline Zhao ZF²2 & 2014 & China & 19 & (1) (2) (3) (4) (5) \\
\hline Shu $X^{23}$ & 2012 & China & 17 & (1) (2) (4) \\
\hline Yao L ${ }^{24}$ & 2012 & China & 12 & (1) (2) (6) \\
\hline$Y_{u} e^{25}$ & 2015 & China & 17 & (1) (2) (3) (5) \\
\hline Lan Y26 & 2011 & China & 32 & (1) (2) \\
\hline
\end{tabular}

Note: (1): abdominal pain; (2): bloating; (3): quality of life; (4): effectiveness; (5): adverse reactions; (6): diarrhea.

Table 1. Summary of the 13 meta-analyses or systematic reviews that qualified for this review.

\subsection{Quality of meta-analyses and systematic reviews}

As shown in Table 2, the AMSTAR score is 5-9 (7.42 \pm 1.22). All documents are of medium quality.

\subsection{Analysis of factors affecting methodology quality}

The stratified analysis of the reported and methodological quality of the 13 studies included showed that the methodological quality of the authors with $\geq 3$ persons was significantly improved, and the difference was statistically significant. However, multiagency cooperation for the methodological quality of the literature helped, but the difference was not statistically significant. See Figure 2.

\section{Discussion}

\subsection{Effectiveness of probiotics in IBS}

IBS is considered a multifactorial disorder associated with visceral hypersensitivity, altered gut motility, and dysfunction of the brain-gut axis and immune system. Recent studies have also shown that patients with IBS exhibited a sustained hypothalamic- pituitary-adrenal (HPA) axis response to acute psychosocial stress; however, the pathophysiology of the disorder is still not completely understood. Of those proposed mechanisms, microbiome change in the gastrointestinal tract has been suggested to play a critical role in the causation and progression of IBS symptoms. ${ }^{27}$ The consumption of probiotic bacteria may increase circulating vitamin $D$,

\begin{tabular}{|c|c|c|c|}
\hline AMSTAR question & Yes & Part & No \\
\hline 1. Was an a priori design provided? & 12 & 1 & 0 \\
\hline $\begin{array}{l}\text { 2. Was a comprehensive literature search } \\
\text { performed? }\end{array}$ & 5 & 5 & 3 \\
\hline 3. Is it possible to replicate the search? & 8 & 5 & 0 \\
\hline $\begin{array}{l}\text { 4. Did the inclusion criteria permit gray } \\
\text { literature? }\end{array}$ & 0 & 10 & 3 \\
\hline $\begin{array}{l}\text { 5. Was a list of studies (included and } \\
\text { excluded) provided? }\end{array}$ & 0 & 0 & 13 \\
\hline $\begin{array}{l}\text { 6. Were the characteristics of the included } \\
\text { studies provided? }\end{array}$ & 13 & 0 & 0 \\
\hline $\begin{array}{l}\text { 7. Was the scientific quality of the included } \\
\text { studies assessed and documented? }\end{array}$ & 8 & 5 & 0 \\
\hline $\begin{array}{l}\text { 8. Were the methods used to combine the } \\
\text { findings of studies appropriate? }\end{array}$ & 13 & 0 & 0 \\
\hline $\begin{array}{l}\text { 9. Was the effect size index chosen justified } \\
\text { statistically? }\end{array}$ & 9 & 4 & 0 \\
\hline $\begin{array}{l}\text { 10. Was the likelihood of publication bias } \\
\text { assessed? }\end{array}$ & 10 & 0 & 3 \\
\hline 11. Was the conflict of interest included? & 6 & 0 & 7 \\
\hline
\end{tabular}

Table 2. Quality of meta-analyses and systematic reviews.

which interacts with the vitamin $\mathrm{D}$ receptor to facilitate an effective immune response. ${ }^{28}$ In one study, ${ }^{18}$ single Bifidobacterium infantis 35624 was not shown to be effective among IBS patients, which might be explained by the fact that numerous probiotics need to work together in order to recover the microbiome in the gastrointestinal tract. It remains challenging to identify most or all probiotic species and strains from the countless gut bacteria. In addition, it is still unclear whether the combination treatment of probiotics and vitamin D supplement could have better efficacy among IBS patients. Among all factors, change in intestinal microbial flora is important in 


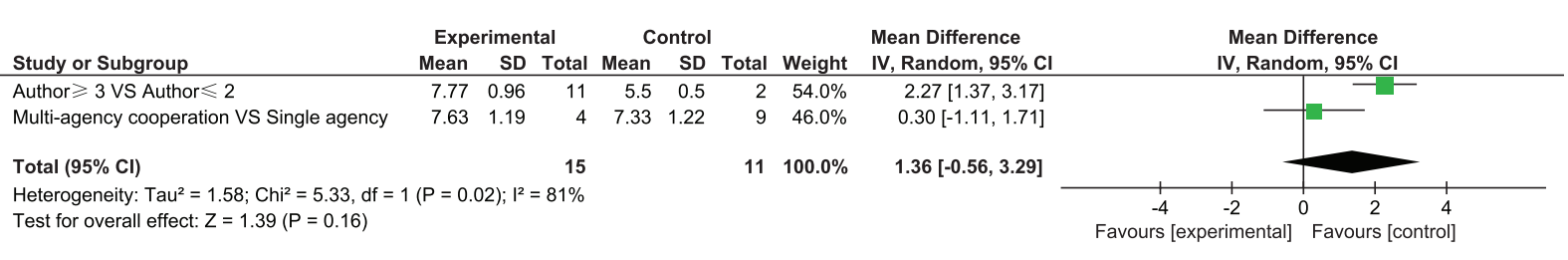

Figure 2. Analysis of the influencing factors of methodological quality of the included studies.

Note: Group A is the group with number of authors $\geq 3$; multi-agency cooperation; Group B is the group with number of authors $\leq 2$; single institution

the initiation of IBS. The use of probiotics in IBS has been confirmed by recovery and gradual healing. Based on the results of the meta-analysis, lactic acid bacteria treatment had effects on children and adults with IBS without any side effects. Additionally, probiotics stabilize immune dysregulation in IBS, thus enhancing cellular integrity to protect the colon. ${ }^{29,30}$ Probiotics also modify the intestinal microbiota, altering the fermentation pattern inside the colon and reducing flatulence. ${ }^{31}$

\subsection{Literature methodology quality evaluation}

This study shows that from the perspective of methodological quality, 13 methodologies are of medium quality. The main problems are: (1) none of the literature provides references to exclude the literature; (2) most of the documents are not retrieved. In order to retrieve the gray literature, the results may be biased; (3) Chinese literature does not explain the relevant conflicts of interest. The four-part literature search strategies are incomplete. A comprehensive search strategy is conducive to improving the recall rate of the literature.

\subsection{Analysis of the factors affecting the quality of the literature}

\subsubsection{Number of authors}

The number of authors $\geq 3$ is better than the number of authors $\leq 2$, the difference is statistically significant. Cochrane Handbook for Systematic Reviews of Interventions requires no less than three people to make a systematic review. The methodological quality evaluation is subjective. When the authors are few, the evaluation of the items only represents the opinions of a few people, and the subjectivity is more obvious, so the difference is statistically significant.

\subsubsection{Institutional cooperation}

Multi-institutional cooperation is superior to a single institution; the difference was not statistically significant. Reason for analysis: Multi-institutional cooperation can provide more manpower and material resources in both the intervention stage and the paper writing stage, so the methodological quality has improved. Multiagency cooperation can complement each other's strengths and jointly promote the development of evidence-based medicine. Therefore, multi-institutional cooperation is recommended.

\subsection{The enlightenment for Chinese systematic review/meta-analysis writing}

To begin with, to train nursing staff to learn the applicable registration system as soon as possible, it is recommended to develop the domestic evidence-based medicine, meta-analysis registration platform, and be in line with international standards. Next, researchers should pay attention to the quality and methodological quality of systematic review, strictly follow the AMSTAR items, and conduct normative and detailed writing. In particular, the analysis of the limitations of evidence intensity and related conflicts of interest should pay attention to the empirical interpretation of the conclusions. Must carefully narrate the research results and make appropriate inferences, rather than eager to make a biased judgment. Finally, due to the limitation of periodical layout and number of words in China, many documents may be deleted before publication, which leads to the fact that their true quality is underestimated. It is therefore suggested that the periodicals establishment of an online reading system provides design plans, search strategies, and all databases. The search formula, the final excluded literature, the content of data statistics, and related charts facilitate the reproduction of the system evaluation.

\section{Limitations and prospects}

Our study has a few limitations. Although we conducted extensive searches, we realized that the keywords and their combinations used to classify systematic reviews and meta-analyses may affect our ability to search without errors. In spite of the supplementary search, it may still have not yet been fully collected, the sample size is limited, there is selectivity bias, and the conclusion is limited. Training and pre-assessment were conducted, 
but because the scale assessment was subjective, it was not ruled out that there were differences in the score results owing to subjective differences in understanding.

Although several reviews and meta-analyses have concluded that probiotics appear to improve overall IBS symptoms, the efficacy of specific probiotic species remains unclear. Despite these findings, some issues concerning the probiotic treatment in IBS patients persist; specifically, the type of probiotic used in different studies varied, combined probiotics and single probiotics were both used, and the doses and treatment durations were also different between studies. Previous meta-analyses usually include numerous or any probiotics in a single review, which adds difficulty to the thorough assessment of a specific species in the symptom relief of IBS patients.

In conclusion, our results demonstrate that probiotic supplementation is an effective therapy in IBS patients. Future studies of the effects of probiotics in IBS should focus on probiotic type, strain, dose, and treatment

\section{References}

1. Wilkins T, Pepitone C, Alex B, Schade RR. Diagnosis and management of IBS in adults. Am Fam Physician. 2012;86:419-426.

2. Spiller R, Aziz Q, Creed F, et al. Guidelines on the irritable bowel syndrome: mechanisms and practical management. Gut. 2007;56:1770-1798.

3. Hulisz D. The burden of illness of irritable bowel syndrome: current challenges and hope for the future. J Manag Care Pharm. 2004;10:299-309.

4. Dupont HL. Review article: evidence for the role of gut microbiota in irritable bowel syndrome and its potential influence on therapeutic targets. Aliment Pharmacol Ther. 2014;39:1033-1042.

5. Öhman L, Törnblom H, Simrén M. Crosstalk at the mucosal border: importance of the gut microenvironment in IBS. Nat Rev Gastroenterol Hepatol. 2015;12:36-49.

6. Hyland NP, Quigley EM, Brint E. Microbiota-host interactions in irritable bowel syndrome: epithelial barrier, immune regulation and brain-gut interactions. World J Gastroenterol. 2014;20:8859-8866.

7. Hussain Z, Quigley EM. Systematic review: complementary and alternative medicine in the irritable bowel syndrome. Aliment Pharmacol Ther. 2006;23:465-471.

8. Jarcho JM, Chang L, Berman M, et al. Neural and psychological predictors of treatment response in irritable bowel syndrome patients with a 5-HT3 receptor antagonist: a pilot study. Aliment Pharmacol Ther. 2008;28:344-352. duration. The quality of the previous meta-analysis methodology was medium, suggesting that meta-analysis reviewers should strictly write in accordance with the AMSTAR criteria and create high-quality meta-analyses to guide clinical practice.

\section{Authors' contributions}

Conceived this editorial: Yuan Jia

Searched and sorted information: Yuan Jia, Li-Min

Guo, Si-Yuan Yang, and Qian Wu

Drafted the manuscript: Yuan Jia

Critically reviewed the whole manuscript: Fan-Jie Meng and Qian Wu

Approved the authorships and submission: Fan-Jie Meng.

\section{Conflicts of interest}

All contributing authors declare no conflicts of interest.

9. Böhn L, Störsrud S, Liljebo T, et al. Diet low in FODMAPs reduces symptoms of irritable bowel syndrome as well as traditional dietary advice: a randomized controlled trial. Gastroenterology. 2015;149:1399-1407. e2.

10. Pimentel M, Lembo A, Chey WD, et al. Rifaximin therapy for patients with irritable bowel syndrome without constipation. N Engl J Med. 2011;364:22-32.

11. Pineiro M, Stanton $C$. Probiotic bacteria: legislative framework - requirements to evidence basis. J Nutr. 2007;137(3 suppl 2):850S-853S

12. Dai $C$, Zheng $C Q$, Jiang $M, M a X Y$, Jiang LJ. Probiotics and irritable bowel syndrome. World J Gastroenterol. 2013;19:5973-5980.

13. Shea BJ, Grimshaw JM, Wells GA, et al. Development of AMSTAR: a measurement tool to assess the methodological quality of systematic reviews. BMC Med Res Methodol. 2007;7:10.

14. Corbitt M, Campagnolo N, Staines D, MarshallGradisnik S. A systematic review of probiotic interventions for gastrointestinal symptoms and irritable bowel syndrome in chronic fatigue syndrome/myalgic encephalomyelitis (CFS/ME). Probiotics Antimicrob Proteins. 2018;10:466-477.

15. Ortiz-Lucas M, Tobías A, Saz P, Sebastián JJ. Effect of probiotic species on irritable bowel syndrome symptoms: a bring up to date meta-analysis. Rev Esp Enferm Dig. 2013;105:19-36.

16. Didari T, Mozaffari S, Nikfar S, Abdollahi M. Effectiveness of probiotics in irritable bowel syndrome: 
updated systematic review with meta-analysis World J Gastroenterol. 2015;21:3072-3084.

17. Zhang Y, Li L, Guo C, et al. Effects of probiotic type, dose and treatment duration on irritable bowel syndrome diagnosed by Rome III criteria: a meta-analysis. BMC Gastroenterol. 2016;16:62.

18. Yuan F, Ni H, Asche CV, Kim M, Walayat S, Ren J. Efficacy of Bifidobacterium infantis 35624 in patients with irritable bowel syndrome: a meta-analysis. Curr Med Res Opin. 2017;33:1191-1197.

19. Ford AC, Quigley EM, Lacy BE, et al. Efficacy of prebiotics, probiotics, and synbiotics in irritable bowel syndrome and chronic idiopathic constipation: systematic review and meta-analysis. Am J Gastroenterol. 2014;109:1547-1561; quiz 1546, 1562.

20. Tiequn B, Guanqun C, Shuo Z. Therapeutic effects of Lactobacillus in treating irritable bowel syndrome: a meta-analysis. Intern Med. 2015;54:243-249.

21. Li DQ. Meta-analysis and evaluation of clinical effects of probiotics on irritable bowel syndrome (IBS). J Pract Diagnosis Ther. 2016;27:4531-4533 (in Chinese).

22. Zhao ZF, Yang T, Yao P. Meta-analysis of the efficacy of probiotics in the treatment of irritable bowel syndrome. Chin J New Drugs Clin Remed. 2014;33:26-35 (in Chinese).

23. Shu XC, Zhou SP, Chen CY, Wang SD, Wang FY. A systematic evaluation of the randomized controlled trial of irritable bowel syndrome with probiotics. Chin J Evidence-Based Med. 2012;12:840-847 (in Chinese).
24. Yao L, Fu L, Zhao SJ. Meta-analysis of the clinical efficacy of probiotics in the treatment of irritable bowel syndrome in China. Chin J Evidence-Based Med. 2012;12:602-607.

25. Hu Y, Tao L, Lyu B. A meta-analysis of probiotics for the treatment of irritable bowel syndrome. Chin $\mathrm{J}$ Internal Med. 2015;54:445-451.

26. Lan Y, Luo HS. A meta-analysis of probiotics in the treatment of irritable bowel syndrome. J Gastroenterol Hepatol. 2011;20:809-813.

27. Salem AE, Singh R, Ayoub YK, Khairy AM, Mullin GE. The gut microbiome and irritable bowel syndrome: state of art review. Arab J Gastroenterol. 2018;19:136-141.

28. Ferguson LR, Laing B, Marlow G, Bishop K. The role of vitamin $D$ in reducing gastrointestinal disease risk and assessment of individual dietary intake needs: focus on genetic and genomic technologies. Mol Nutr Food Res. 2016;60:119-133.

29. Martínez C, González-Castro A, Vicario M, Santos J. Cellular and molecular basis of intestinal barrier dysfunction in the irritable bowel syndrome. Gut Liver. 2012;6:305-315.

30. Major G, Spiller R. Irritable bowel syndrome, inflammatory bowel disease and the microbiome. Curr Opin Endocrinol Diabetes Obes. 2014;21:15-21.

31. Jiang T, Savaiano DA. Modification of colonic fermentation by bifidobacteria and $\mathrm{pH}$ in vitro. Impact on lactose metabolism, short-chain fatty acid, and lactate production. Dig Dis Sci. 1997;42:2370-2377. 